\title{
MINEUR JUSQU'AUX OS ? LA JUGE DES ENFANTS ET L'ÂGE DU JEUNE ÉTRANGER AU PRISME DES TESTS OSSEUX
}

\author{
$\underline{\text { Annalisa Lendaro }}$
}

Presses Universitaires de France | «Ethnologie française »

2020/2 N 178 | pages 377 à 389

ISSN 0046-2616

Article disponible en ligne à l'adresse :

https://www.cairn.info/revue-ethnologie-francaise-2020-2-page-377.htm

Distribution électronique Cairn.info pour Presses Universitaires de France.

(C) Presses Universitaires de France. Tous droits réservés pour tous pays.

La reproduction ou représentation de cet article, notamment par photocopie, n'est autorisée que dans les limites des conditions générales d'utilisation du site ou, le cas échéant, des conditions générales de la licence souscrite par votre établissement. Toute autre reproduction ou représentation, en tout ou partie, sous quelque forme et de quelque manière que ce soit, est interdite sauf accord préalable et écrit de l'éditeur, en dehors des cas prévus par la législation en vigueur en France. Il est précisé que son stockage dans une base de données est également interdit. 


\title{
Mineur jusqu'aux os? \\ La juge des enfants et l'âge du jeune étranger au prisme des tests osseux
}

\author{
Annalisa Lendaro \\ CNRS, Centre d'étude et de recherche Travail Organisations Pouvoir (UMR 5044) \\ annalisa.lendaro@univ-tlse2.fr
}

\section{RÉSUMÉ}

Cette contribution se propose d'analyser la place des tests osseux dans le processus de (non)reconnaissance de la minorité du jeune étranger isolé. En particulier, l'article se focalisera sur les débats concernant la capacité de ces derniers à "dire vrai " au sujet de l'âge des jeunes, qui ont lieu pendant et en marge des audiences devant le juge des enfants. S'appuyant sur les matériaux recueillis via une enquête ethnographique réalisée dans deux villes françaises, le cœur de la contribution analyse les débats autour de la légitimité et de la fiabilité de ces «technologies du soupçon» et met en exergue la dimension politique des pratiques judiciaires autour des expertises sur le corps d'autrui.

Mots clés : Mineurs non accompagnés. Expertises osseuses. Détermination de l'âge. Juge des enfants. Technologies du soupçon.

\section{- La fabrique d'un "âge vraisemblable": le jeune étranger et la juge}

À l'automne 2017, Issa et Adama ${ }^{1}$ arrivent par le train dans la ville où je réside à l'époque. L'un est Malien, l'autre vient de Guinée Conakry. N'ayant pas de contacts sur place, ils dorment plusieurs nuits dans la rue, à proximité de la gare, avant d'être repérés par les membres d'un collectif qui est à l'initiative d'un réseau d'hébergeurs solidaires. Pendant une semaine environ, nous partageons le quotidien et des bribes de nos histoires respectives. Issa et Adama décident de se présenter au dispositif d'évaluation des mineurs isolés (DEMI) qui, ayant décrété leur majorité au bout de cinq jours de mise à l'abri, leur communique oralement qu'ils ne seront pas pris en charge par l'Aide sociale à l'enfance (ASE), et qu'ils peuvent faire appel de cette décision via un avocat. "ASE ", "conseil départemental ", «appel»: Issa et Adama ne comprennent pas ce que la personne qui les a évalués leur dit, à part qu'ils sont de nouveau à la rue. Quelques semaines plus tard, un bâtiment inoccupé est réquisitionné par une cinquantaine de jeunes et de militant'e-s. Les deux adolescents s'y installent, et nous gardons contact. Petit à petit, de nouveaux éléments de leur histoire émergent, souvent sous la forme d'interrogations : «Tu connais cette avocate? - Non. - Elle m'a dit que la juge pourrait me demander d'aller à l'hôpital pour un examen. - Quel examen? - Je ne sais pas".

C'est dans ce contexte que ma recherche sur le contentieux des Mineurs non accompagnés (MNA) et sur la place des tests osseux dans le processus de détermination de l'âge prend forme. Ce sont les interactions avec des jeunes désemparés par l'incertitude de procédures dont ils ne connaissent pas toujours les enjeux et les risques, épuisés par l'instabilité de ces hébergements toujours temporaires, et avec les avocates ${ }^{2}$ qui les assistent, qui font converger mes questionnements vers le processus de fabrication de l'âge d'un jeune, dont le corps est parfois appelé à témoigner, ou plus précisément à servir d'indice. 


\section{Encadré 1. Matériaux et méthodologie}

Depuis 2016, je suis les actions promues par un collectif d'hébergeurs et de militant.e.s qui accompagne les jeunes exilés dans une ville du sud-ouest de la France. Depuis 2017, je recueille (de manière formelle, mais souvent via des échanges plus informels et spontanés) des récits de vie de jeunes, des témoignages d'hébergeurs, et des avocates qui défendent leur cause. À partir de 2018, j'ai élargi cette recherche aux juges des enfants de deux tribunaux de grande instance (ici, TGI 1 et TGI 2) et aux professionnels de l'évaluation (un médecin légiste et les agents du DEMI). Cette enquête ethnographique a donc lieu dans un milieu d'interconnaissance forte, caractérisé par des enjeux personnels et réputationnels qui traversent les espaces étudiés, et elle implique de combiner plusieurs outils qualitatifs de recueil des données (observations, entretiens compréhensifs, récits de vie, archives et documents officiels des avocates et des greffes du TGI). Les matériaux recueillis sont issus de : huit entretiens avec des avocates défendant les MNA dans les deux départements (ici, D1 et D2), cinq entretiens avec des juges s'occupant du contentieux MNA, huit entretiens avec des jeunes et six entretiens avec des hébergeurs et militant.e.s (ainsi que de nombreuses discussions informelles), un entretien avec un médecin légiste, un entretien avec un informateur travaillant au conseil départemental, deux entretiens avec des agents du DEMI, les notes d'observation de treize audiences ${ }^{3}$, les notes prises lors de cinq rendez-vous de préparation d'audience entre une avocate et ses clients, la constitution et l'analyse d'une base documentaire concernant les expertises osseuses (composée à la fois de la littérature scientifique et médicale, d'articles de presse, de rapports d'associations et de collectifs), un corpus de 51 décisions de justice (41 en première instance, 8 à la cour d'appel, et 2 en cassation, fournies par les avocates) et d'une vingtaine d'expertises osseuses, respectivement rendues et réalisées entre 2016 et 2019 (fournies par les avocates une fois anonymisées, elles faisaient partie des dossiers de leurs clients).

Comment se fabriquent l'" âge vraisemblable » et le " récit crédible » d'un jeune étranger se disant mineur, et quelle est la place des tests osseux dans ce processus largement judiciarisé d'évaluation? Autrement dit, le corps peut-il contribuer à " dire vrai » au sujet de l'âge, voire témoigner de la "bonne foi " d'un individu et de la crédibilité de son discours [Fassin, Bouagga et Coutant, 2013] ? Des économies morales ${ }^{4}$ conflictuelles autour de ces expertises apparaissent en filigrane : que nous dit le recours à ces tests sur une société qui investit les corps comme régime de preuve? Qu'est-ce que le contentieux des MNA permet de montrer au sujet de ces tests? Sont-ils censés "dire vrai » au sujet de l'âge? Quelle est la dimension politique et morale des luttes judiciaires autour des expertises sur le corps d'autrui ?

Si cette procédure de détermination d'un " âge vraisemblable " s'applique à l'ensemble des jeunes étrangers qui se disent mineurs et isolés, c'est bien parce que cette population est soupçonnée de fraude. Autrement dit, la méfiance vis-à-vis du discours et le doute autour de l'authenticité des documents d'état civil des jeunes étrangers justifie le recours à des techniques de "biologisation de la vérité " qui transforment le corps en preuve [Gourarier, 2017 ; Dodier, 1991 et 1993] ou, a minima, en indice, selon la fiabilité que la juge décidera de leur accorder lors de l'exercice de son pouvoir souverain d'appréciation.

S'inspirant d'une riche production scientifique autour de la construction sociale et politique de l'âge [Prout, 2000 ; Diasio, 2014 et 2015 ; Diasio et Vinel, 2017], ce texte apporte un éclairage sur les usages des expertises médicales sur le corps de jeunes étrangers considérés tantôt en danger, tantôt dangereux (notamment pour les finances publiques) ${ }^{5}$.

Après avoir présenté le contexte politique et juridique, ainsi que les controverses scientifiques autour de la fiabilité des tests osseux, l'article aborde la question de la légitimité et de la fiabilité (accordées ou contestées, selon la juge des enfants) de ces «technologies du soupçon » [Collectif Cette France-là, 2010] : quelles pratiques, quelles justifications, et quelles visions de la justice s'affrontent au prisme des interactions entre les JE et les jeunes, leurs avocates, et le conseil départemental ? L'article montre que ce processus judiciarisé de construction de la (non)minorité est traversé par des tensions politiques et morales d'autant plus fortes que la fiabilité des techniques de biologisation de l'âge est contestée.

\section{Enjeux politiques et juridiques autour \\ de la reconnaissance de la minorité des étrangers}

Selon de nombreuses institutions, telle que la Mission mineurs non accompagnés (M-MNA) relevant du ministère de la Justice, une augmentation importante du nombre d'arrivées de jeunes étrangers aurait progressivement bousculé le dispositif d'accueil français : le dernier rapport de cet organisme ${ }^{6}$ parle de 17022 personnes se déclarant MNA en 2018, contre 14908 en 2017, 8054 en 2016, et 2555 en $2013^{7}$. Un autre rapport récent, rédigé au nom de la commission des affaires sociales du Sénat ${ }^{8}$, parle d'une problématique dont l'ampleur "ne cesse de croître de 
manière spectaculaire " [Rapport d'information $\mathrm{n}^{\circ} 598$ : 11], et fournit des données selon lesquelles les MNA pris en charge par les conseils départementaux auraient été 13000 en 2016 et 25000 fin 2017 [ibid. : 11]. À son tour, l'Assemblée des départements de France (ADF) estimait à 54000 le nombre d'évaluations de minorité effectuées en 2017, et à 40000 le nombre de prises en charge pour $2018^{9}$.

$\mathrm{La}$ référence récurrente à une "explosion du nombre d'arrivées" [ibid. : 11], à une "croissance exponentielle du nombre des MNA» [Rapport d'information $\mathrm{n}^{\circ} 598: 23$ ], à la responsabilité de «filières qui connaissent très bien le fonctionnement de l'aide sociale à l'enfance en France " [ibid. : 26] ou encore à la nécessité de transférer la charge de l'évaluation et de la mise à l'abri à l'État ${ }^{10}$, témoignent de la mise sur agenda de la question des MNA en tant que problème public [Hassenteufel, 2010]. Le Sénat, au sujet du projet de loi de finance 2019, parle explicitement d'un "accroissement constant de l'arrivée sur le territoire national de mineurs non accompagnés (MNA)", qui «pose un problème financier inédit " ${ }^{11}$.

La détermination d'un âge inférieur ou supérieur à 18 ans attribuable au jeune non européen est centrale : en effet, la reconnaissance de sa minorité entraîne l'obligation pour le conseil départemental de le prendre en charge ${ }^{12}$ et interdit son éloignement du territoire, alors qu'à l'inverse, sa majorité n'ouvre aucun droit et implique un risque réel de faire l'objet d'une obligation de quitter le territoire français (OQTF) voire d'être poursuivi pénalement pour usage de faux. De ce fait, le processus de détermination de la (non)minorité est encadré par une procédure très institutionnalisée (mais pas homogène pour autant dans sa mise en œuvre sur le territoire national) dont la charge revient aux départements et à l'Aide sociale à l'enfance (ASE), aux prises avec le souci principal de limiter ses coûts [Carayon, Mattiussi et Vuattoux, 2018].

Malgré ce constat, les données statistiques montrant la progressive baisse de reconnaissance de minorité ont tendance à être interprétées comme une preuve des comportements frauduleux des jeunes et non pas comme la conséquence de la volonté de contingenter leur accueil [Bailleul 2017]. Le soupçon semble donc peser bien plus lourd que la présomption de minorité le long du processus d'évaluation dont ces jeunes étrangers font l'objet, et ce à n'importe quelle étape de la procédure d'évaluation [Carayon, Mattiussi et Vuattoux, 2020]. Cette dernière, en partie définie par la loi du 14 mars 2016 et ensuite par l'arrêté du 17 novembre 2016, vise à permettre le recueil d'un "faisceau d'indices" dont différents opérateurs de jugement [Perrot, 2016] se serviront pour rendre, chacun leur tour, un avis sur la minorité ou la majorité du jeune étranger, avant que l'autorité judiciaire ordonne son placement ou bien conclue à un non-lieu à assistance éducative.

\section{Repères juridiques et procéduraux autour du processus d'évaluation}

Les agents du DEMI ${ }^{13}$, sous la responsabilité du conseil départemental (CD), interviennent en premier : pendant la toute première phase de mise à l'abri dont la durée fixée par la loi est de cinq jours, ils sont censés interroger et observer le jeune se déclarant mineur ; ils doivent ensuite transmettre une synthèse de leur rapport au Président du CD indiquant clairement l'avis autour de la minorité du jeune ${ }^{14}$. Dans le but, pour le moins ambitieux, de limiter la nature arbitraire du processus d'évaluation, l'arrêté interministériel du 17 novembre 2016 fixe un référentiel national des pratiques. Six points doivent être abordés pendant les entretiens, conduisant "à la rédaction d'un rapport de synthèse concluant ou non à la qualité de mineur » :

1) l'état civil : le jeune est invité à présenter sa situation personnelle, son état civil, son pays et région d'origine, et à fournir tout document d'état civil en sa possession puis à en préciser les conditions d'obtention. Il est intéressant de noter que ce point rentre en conflit avec le principe de présomption d'authenticité des actes d'état civil émanant d'une administration étrangère inscrit dans l'article $47 \mathrm{du}$ Code civil, notamment en ce que l'arrêté précise que l'évaluateur est appelé à constater les éventuelles "incohérences entre le document présenté et le récit de la personne ", et qu'il peut donc demander "des précisions" au jeune, et l'indiquer dans le rapport de synthèse ;

2) la composition familiale : le jeune est questionné sur la composition de sa famille, sur ses proches, sur l'identité et l'âge de ses parents et sur sa fratrie, les liens qu'il entretient avec toutes ces personnes;

3) les conditions de vie dans le pays d'origine, notamment en matière de conflits, de situation économique, de scolarisation, d'activité professionnelle au pays ;

4) les motifs de départ et la présentation du parcours migratoire, le financement du voyage vers l'Europe, les étapes principales, les événements marquants ;

5) les conditions de vie depuis l'arrivée en France, i.e. la date d'entrée sur le territoire, le type d'hébergement s'il a lieu, les soutiens etc. ; 
6) le projet personnel, en lien notamment avec la scolarité et l'éventuelle demande d'asile.

En même temps qu'il précise les questions qui doivent être abordées pendant le ou les entretiens entre l'évaluateur et le jeune qui se dit mineur, l'arrêté propose de limiter la place de considérations subjectives dans un domaine où les certitudes sont peu nombreuses, et où le doute guide le travail des professionnels. En effet, ces derniers sont tenus de "confronter l'apparence physique de la personne évaluée, son comportement, sa capacité à être indépendante et autonome, sa capacité à raisonner et à comprendre les questions posées, avec l'âge qu'elle allègue " (art. 5), sans pour autant pouvoir déterminer de manière irréfutable quels sont les signes d'indépendance qui peuvent confirmer la majorité, ni inversement les comportements qui témoignent d'un âge inférieur à dix-huit ans. Selon l'agent donc, l'évaluation peut conduire à un refus de prise en charge pour majorité supposée dès lors que le jeune se démontre suffisamment autonome pour se préparer tout seul à manger.

Souvent, les agents du DEMI ne sont pas en mesure de produire un avis motivé dans les temps impartis. C'est notamment le cas lorsque ces professionnels du social ont un doute quant à l'authenticité des documents d'état civil fournis par le jeune. Ils peuvent alors le souligner dans leur rapport au CD et ce dernier peut demander qu'une expertise documentaire soit réalisée : en effet, la circulaire interministérielle du 25 janvier 2016 prévoit qu' " afin de contribuer à cette évaluation, les préfectures de département, saisies par le conseil départemental, apporteront, par la mobilisation des compétences des services de l'État, une expertise en matière de fraude documentaire ". Une fois le rapport reçu, le président du CD a trois options : il peut transmettre les documents d'identification de la personne aux services chargés de la lutte contre la fraude documentaire de la PAF, si un doute quant à leur validité a été émis par l'évaluateur ; il peut saisir l'autorité judiciaire, soit aux fins d'assistance éducative (lorsque la personne est évaluée mineure), soit afin de procéder aux investigations complémentaires (notamment les tests osseux ${ }^{15}$ ) selon les conditions posées à l'article 388 du Code civil (art. 8 AI 17 novembre 2016) ; ou alors il peut notifier à la personne une décision motivée de refus de prise en charge, en précisant quelles sont les voies de recours applicables (art. 9 AI 17 novembre 2016). Il est possible alors pour le jeune de formuler, via son avocate, un recours auprès de la juge des enfants (avec des temporalités parfois longues qui peuvent le mettre en danger, notamment s'il se retrouve à la rue). Lorsque le contentieux finit en appel, le jeune a parfois passé plusieurs mois en errance.

D'un point de vue juridique et procédural, il apparait donc que les éléments faisant fonction d'indices au sujet de l'âge du jeune sont multiples et qu'ils entretiennent des liens d'interdépendance : tout d'abord, il faut considérer les documents d'état civil, ensuite le rapport du DEMI, et si jamais des doutes subsistent (notamment parce qu'un avis ${ }^{16}$ défavorable a été émis par le service de la PAF sur les papiers) l'autorité judiciaire peut ordonner les tests osseux.

Il apparaît également que le subjectif et l'arbitraire sont destinés à jouer un rôle important pendant l'évaluation et au cours de la procédure judiciaire. L'appréciation du procureur et de la juge des enfants est notamment centrale car ce sont eux qui sont censés reconnaître la primauté des actes d'état civil étrangers, et déterminer si et pourquoi les expertises osseuses peuvent être ordonnées. L'article 388 du code civil prévoit en effet que : "Les examens radiologiques osseux aux fins de détermination de l'âge, en l'absence de documents d'identité valables et lorsque l'âge allégué n'est pas vraisemblable, ne peuvent être réalisés que sur décision de l'autorité judiciaire et après recueil de l'accord de l'intéressé. Les conclusions de ces examens, qui doivent préciser la marge d'erreur, ne peuvent à elles seules permettre de déterminer si l'intéressé est mineur. Le doute profite à l'intéressé.» Au moins deux principes essentiels sont ici défendus. Le premier fixe une double condition à l'ordonnancement des tests osseux : la certitude de la non validité des documents, et le sentiment, très subjectif, que l'âge allégué par le jeune est invraisemblable. Selon le deuxième principe, si l'un des deux critères n'est pas rempli, la présomption de minorité devrait prévaloir lors de la décision.

Pourquoi donc certains juges des enfants ordonnent-ils des tests osseux alors que les documents d'identité sont considérés comme authentiques? Pourquoi d'autres ne le font-ils pas ? L'enquête réalisée dans deux départements du sud de la France révèle que le doute sur l'âge allégué par le jeune étranger traverse l'ensemble des phases de son évaluation, allant souvent jusqu'à vider d'effectivité le principe de présomption de minorité. Selon leur interprétation des textes et de la jurisprudence, les juges auront tendance à considérer les expertises médicales en tant qu'indices plus ou moins utiles dans l'enquête autour de l'âge d'une personne. 


\section{Encadré 2 : Controverses scientifiques autour des tests osseux}

Pourquoi est-il si difficile de déterminer de manière irréfutable l'âge d'une personne? Tout d'abord, parce que le terme d'« âge » renvoie à des chronologies différentes. Ainsi l'âge réel, dit chronologique, fait référence à la durée écoulée entre la naissance d'une personne et une date donnée. Les expertises médico-légales requises par les autorités judiciaires tentent précisément de se rapprocher de cet âge chronologique. Le problème naît du peu d'outils fiables pour le faire en l'absence de certitudes sur l'âge civil, ou âge administratif, qui est déterminé par les documents d'identité que la personne possède. Ce sur quoi ces expertises médico-légales sont plus à même de s'exprimer, c'est l'âge biologique, qui se réfère à l'état de vieillissement d'une personne, et qui résulte de la prise en compte de tous les paramètres biologiques et physiologiques au sujet d'un individu. L'appréciation globale du degré de vieillissement biologique ne saurait se faire autrement que par un complexe processus multifactoriel, fondé sur la combinaison de méthodes morphologiques et morphométriques [Saint-Martin, 2014]. La détermination conjointe de l'âge osseux ${ }^{17}$, de l'âge dentaire et de l'âge pubertaire permet l'établissement de l'âge biologique. Or, les tests osseux que les autorités judiciaires peuvent ordonner lorsque le soupçon pèse à la fois sur les documents d'état civil et sur le récit d'un individu se déclarant mineur non accompagné, se résument à une radiographie de la main et du poignet gauche ou, plus récemment, à un scanner de la clavicule. Ces tests sont extrêmement controversés. Tout d'abord, leur validité scientifique, précisément leur «capacité à dire vrai » au sujet de l'âge d'un jeune, est remise en question par les autorités médicales, juridiques et éthiques ${ }^{18}$. Une vaste littérature est désormais disponible sur ce sujet [Pruvost, Boraud et Chariot, 2010 ; Plate-forme Mineurs en Exil, 2017], et la critique consistant à dire que la marge d'erreur (entre 18 mois et 3 ans) doit être prise en compte est généralement admise par les juges des enfants de D1 et D2. Du moins pour ce qui est du test qui a été le plus pratiqué jusque-là : la radiographie de la main et du poignet gauche. Le médecin légiste, via un logiciel, compare cette radiographie à un atlas composé de plusieurs radiographies de ces mêmes parties du corps. Les radiographies de référence sont répertoriées dans un atlas, dit « de Greulich et Pyle » du nom des chercheurs qui l'ont réalisé dans les années 1950 [Greulich et Pyle, 1959]. Leur but était uniquement de permettre une estimation des éventuels retards de croissance, et nullement de déterminer l'âge chronologique des sujets étudiés. Outre le détournement de son usage initial à des fin judiciaires, l'usage contemporain de l'échantillon de l'atlas fait l'objet de nombreuses réserves, notamment parce qu'il se compose d'une série de radiographies appartenant à des enfants et adolescents blancs et de classe moyenne recueillies dans les années 1930. Ainsi, le Haut Conseil de la santé publique, dans son rapport du 23 janvier 2014, précise que " la maturation d'un individu diffère suivant son sexe, son origine ethnique ou géographique, son état nutritionnel ou son statut économique ». Pour la Société francophone d'imagerie pédiatrique, qui a vérifié la validité des images en question à partir d'autres échantillons, l'atlas est toujours valable pour une population européenne et canadienne, mais "une imprécision incompressible doit être prise en compte quand l'atlas est utilisé dans un cadre médico-légal ». D'autres études médicales ont mis en évidence l'inadéquation de ces «images témoin » lorsqu'elles sont appliquées à une population sub-saharienne ou encore marocaine : l'âge chronologique était surestimé jusqu'à trois ans lors de l'utilisation de l'atlas [Ontell et al., 1996]. Ces études s'accordent sur le fait que les paramètres socio-économiques, nutritionnels, géographiques, traumatiques, environnementaux, et de genre jouent un rôle important dans le développement osseux des individus, rendant la référence à l'atlas injustifiée du point de vue scientifique [Noll, 2016]. La deuxième technique la plus utilisée en France pour la détermination de l'âge osseux est le scanner de la clavicule. La littérature scientifique disponible sur le sujet montre que, lorsque l'épiphyse de la clavicule est complètement fusionnée, les individus de sexe féminin ont au moins vingt ans, et ceux de sexe masculin au moins vingt et un ans [Hermetet et al., 2018]. Le recours à ces tests dans le cadre du contentieux des MNA a donc pour finalité d'exclure de la prise en charge au moins les personnes dont l'âge serait vraisemblablement supérieur à 20 ans, plutôt qu'à tenter de déterminer leur âge chronologique. Les problèmes de fiabilité des résultats sont cependant similaires à ceux que posent les tests radiologiques de la main en ce que les populations de référence de ces études (Allemands et Australiens) ne sont pas représentatives des populations de MNA examinées dans les procédures judiciaires en France, et que les données analysées ne prennent pas en compte le poids de variables telles l'origine sociale, l'environnement, les habitudes alimentaires, le parcours individuel et les éventuels traumatismes. D'un point de vue éthique, le recours à des examens radiologiques qui exposent la personne à des rayons ionisant (en proportion exponentiellement plus importante dans le cas du scanner de la clavicule) pose question notamment parce que la procédure n'est pas incluse dans un protocole clinique [Focardi et al., 2014], c'est-à-dire réalisée à des fins médicales ${ }^{19}$. En outre, la question du consentement se pose de façon récurrente, car si la personne doit théoriquement avoir reçu tout un ensemble d'informations au sujet des finalités du test, ses contre-indications et les risques inhérents avant de donner son accord, la pratique contredit largement ces dispositions. En effet l'on sait désormais, par les rapports d'associations [Médecins du monde, 2017 et 2019 ; GISTI, 2017 ; Humans Rights Watch, 2018] mais aussi la restitution d'enquêtes scientifiques [Bailleul et Senovilla Hernandez, 2016], que très souvent la compréhension des enjeux du test est compromise par l'absence d'interprète, mais surtout que le refus de consentement a pu porter préjudice aux jeunes car certains magistrats en ont fait un indice de majorité. L'effet dissuasif de ces décisions de justice doit donc être pris en compte lorsque l'on se penche sur les débats éthiques que les conditions de réalisation de ces examens soulèvent. Dans le basculement de l'univers de la santé à la sphère judicaire [Chariot, 2010], l'examen clinique voit sa nature changer : l'expertise sur le corps d'autrui devient un indice parmi d'autres de la minorité ou de la majorité (ne pouvant pas 
en faire la preuve pour autant à lui seul), et l'estimation de la maturation osseuse est détournée de ses finalités médicales initiales au profit de la détermination de l'âge chronologique du sujet [Lamarche-Vadel, 2016]. L'institutionnalisation de ces "technologies du soupçon" [Gourarier, 2017 ; Vailly et Krikorian, 2018] fait passer le sujet d'un statut théorique de patient à celui de fraudeur potentiel, que l'on peut exposer à des rayons ionisants sans raisons médicales et sans son réel consentement. À ces réserves d'ordre scientifique et éthique, il faut rajouter des pratiques professionnelles variables, tant au niveau de l'autorité judiciaire, qui peut ordonner plus ou moins fréquemment ces tests selon les départements, qu'au niveau des unités médico-judicaires (UMJ) dont les conventions en termes d'exécution des examens et de rédaction des résultats peuvent varier sensiblement [IGA et al., 2018].

\section{- Les "technologies du soupçon" à l'épreuve des pratiques judiciaires locales}

Une des raisons de se méfier des statistiques montrant l'écart entre le nombre de MNA évalués et le nombre de mineurs effectivement pris en charge sur une année (environ la moitié) ${ }^{20}$, tient au fait que ce taux ne tient pas compte des décisions prises par les juges des enfants après une saisine directe par le jeune à la suite d'un refus du DEMI. Le Conseil national des barreaux (CNB) a estimé que sur l'ensemble des recours examinés en 2016 et 2017 à Paris, où les jugements sont pourtant plutôt défavorables par rapport à la moyenne nationale, un jugement sur deux a invalidé l'évaluation initiale du DEMI ayant conclu à la majorité, et a donc enjoint à l'ASE de prendre en charge le jeune.

Le nombre de saisines directes des juges des enfants après un premier refus d'admission à l'ASE a fortement augmenté dans les deux départements sur lesquels cette enquête s'est concentrée. L'augmentation de ce contentieux a impacté directement la charge de travail mais aussi les habitudes professionnelles des JE :

C'est un contentieux difficile. Depuis septembre [2018] on a essayé de partager le contentieux entre plusieurs juges pour que... justement, ce soit un peu moins lourd pour une personne parce que... il y a un côté très compliqué parce que à la fois on voudrait toutes je pense faire le mieux possible, individualiser les choses et tout, mais bon la réalité c'est que... on enchaîne les dossiers... qu'on le veuille ou non, les jeunes portent tous un nom un peu semblable... on trouve un peu des fois qu'ils ont la même tête... on a beaucoup de mal par rapport aux autres audiences (des mineurs non étrangers NDR) où l'on est très impliquées sur des dossiers à individualiser... Parce qu'il y a un truc très difficile à vivre dans ce contentieux, c'est qu'on a l'impression de faire des trucs à la chaîne. (JE 1, cheffe de cabinet au tribunal des enfants, TGI 1)

Au TGI 1 et 2, les jeunes dont les JE doivent évaluer l'âge sont très majoritairement d'origine subsaharienne. La verbalisation d'une difficulté à personnaliser les dossiers à cause d'une supposée ressemblance physique ("ils ont la même tête ») fait apparaitre un processus de construction racialisée de l'âge du jeune qui efface ses caractéristiques individuelles au profit d'une assimilation aux caractéristiques d'un groupe [Barrett et George, 2005]. Aussi, l'augmentation du nombre de dossiers à traiter va de pair avec le sentiment de devoir faire un travail qui s'éloigne de la sphère d'action principale du JE, traditionnellement appelé à protéger des enfants victimes de violence, ressortissants nationaux, dont la prise en charge par l'ASE implique un travail et un suivi dans le temps avec le jeune et les détenteurs de l'autorité parentale :

Bon, je vais vous dire franchement que le contentieux MNA, ça ne fait pas rêver les JE. Parce qu'on est quand même loin de notre cœur de métier. On est très loin de l'enfance maltraitée. Là, on applique presque bêtement les règles de droit qui à mon avis ne sont pas du tout adaptées à la situation. Ce sont des mineurs qu'on ne revoit pas. C'est presque une caisse d'enregistrement. Comme métier, juge des enfants c'est pas ça. C'est revoir les familles, voir évoluer des situations, se fixer des objectifs. (JE 2, cheffe de cabinet au tribunal des enfants, TGI 2)

En outre, les tensions sont nombreuses autour du contentieux des MNA, et les juges font état d'un travail sous pression. "Au sujet des MNA il y a une forte pression, une forte pression de l'ASE, qui dit : "N'ouvrez pas trop les vannes". Parce qu'ils n'ont pas forcément les moyens financiers et voilà " (JE 3, TGI 1). Les conseils départementaux peuvent, notamment à l'occasion de réunions organisées périodiquement entre les JE, le DEMI et l'ASE de chaque département, exercer des pressions directes et personnalisées vis-à-vis des juges qui adoptent des pratiques 
considérées trop favorables aux jeunes et donc préjudiciables pour le conseil départemental.

En réunion au conseil départemental, il y a un mois, les yeux étaient braqués sur moi, en me disant : "Bon estce que vous pouvez vous expliquer là-dessus ? On avait mis en place des protocoles que vous ne respectez pas ". Bon voilà. Après le service du DEMI a pu me dire que, à la même occasion... me dire : "Oui, pourquoi vous ne faites pas plus d'expertises osseuses ?». [...] Donc bon voilà, et du coup je leur ai dit : "Il faudrait vraiment que j'aie suffisamment de doutes ». Et tout de suite, je n'ai pas eu le temps de finir ma phrase qu'on m'a dit : "Mais nos rapports ne suffisent pas pour que vous ayez un doute sur la minorité ?»(JE 3, TGI 1)

Ainsi, ces réunions de concertation entre magistrats, représentants du DEMI, et du conseil départemental deviennent des espaces où se négocient en creux des protocoles de conduite autour de l'évaluation de l'âge, qui sont autant d'occasions pour les participants de faire valoir leur point de vue, d'affirmer la légitimité de leurs choix, ou de contester et marginaliser les pratiques d'autres acteurs. C'est ainsi que "ne pas avoir assez de doutes » et donc ordonner trop de placements à l'ASE est un comportement reproché par le DEMI à la JE 3.

Tiraillées entre des injonctions antagonistes, les JE ont le sentiment que leurs décisions, quelles qu'elles soient, feront l'objet de critiques :

Tout le monde n'est jamais content. Tantôt on vous reproche de trop placer, tantôt on vous reproche de ne pas placer alors qu'on devrait. Avec parfois des décisions de la Cour d'appel qui viennent ne pas confirmer les décisions des JE. Bon. Ce qui est normal. Enfin, la cour n'est pas là pour dire forcément comme nous, mais bon, c'est quand même ressenti comme... Bon ben finalement personne n'est content. Même la cour d'appel. (JE 1, TGI 1)

En plus de la pression exercée par les institutions, d'autres difficultés compliquent le travail des magistrats. Certaines mettent l'accent sur la dimension immorale de l'injonction que l'État fait aux juges de participer au travail de sélection des jeunes :

Le problème il est quand même vraiment là. On nous met dans une position de tri qui est insupportable. C'est pour ça que ce contentieux est difficile à supporter. Parce que, sauf si l'on dit, mais c'est rare je pense, «on s'en fout, ils n'avaient qu'à pas venir ", personne n'est insensible à ça, quand les gens sont dans la rue. On nous fait jouer un rôle qui est très très compliqué. (JE 1, TGI 1)

Si ce travail est difficile, et parfois moralement répréhensible selon certaines juges, c'est aussi parce qu'elles considèrent que la loi ne fournit pas assez d'outils pour travailler correctement, autrement dit, pour guider les magistrates dans cette estimation de l'âge sans devoir faire appel à des impressions, à un sentiment (de "véracité du récit", d'apparence physique, etc.) :

La loi, elle dit clairement que les mineurs, on les protège, et que pour les autres aujourd'hui, il n'y a pas de droit au séjour. Sauf que la loi ne nous aide pas en ce qu'elle ne dit pas comment on fait pour savoir si une personne est mineure, alors qu'on sait que la plupart de ces personnes viennent de pays où l'état civil n'est pas fiable, de zones de grande difficulté, de pauvreté, voilà, et qu'il y ait la fraude c'est une réalité. (JE 1, TGI 1)

Les textes sont même d'autant plus critiqués que les magistrates sont persuadées de la récurrence de comportements frauduleux de la part des jeunes :

Pour l'instant, l'on admet qu'à partir du moment où une personne se présente avec un acte d'état civil qui n'est pas falsifié, on doit considérer qu'il lui est rattachable. En sachant qu'on sait très bien qu'en Afrique, sur les marchés, vous achetez un acte de naissance comme vous achetez un poulet. [...] Bah... la plupart des jeunes sont majeurs bien évidemment, ça on le sait, mais moi j'estime que si on ne peut pas écrire qu'ils sont majeurs, je dois le considérer comme mineur parce que ça, c'est la loi, je ne peux pas faire autrement. (JE 2, TGI 2)

Autrement dit, face à la fraude généralisée dont elles sont convaincues d'être les témoins, plusieurs juges estiment manquer d'instruments juridiques pour exclure la prise en charge pour les jeunes qu'elles croient majeurs, faute de preuves irréfutables de leur majorité. De fait, l'ensemble des JE pensent disposer de peu d'outils d'appréciation "objectifs " de l'âge, à l'exception des vérifications documentaires qu'elles sous-traitent à la PAF et éventuellement des examens osseux qu'elles peuvent ordonner aux unités médicojudiciaires (UMJ).

\section{Les tests osseux: les arbitrages des juges des enfants}

Le recours aux tests osseux par les JE dans les deux départements étudiés a fortement augmenté depuis 
2016. Alors que sur les cinq juges interrogées, qui représentent l'ensemble des JE s'occupant du contentieux MNA au TGI 1 et 2, trois d'entre elles disent y recourir a minima et le cas échéant ne pas accorder beaucoup de poids aux résultats de ces expertises, les décisions de justice examinées entre 2016 et début 2019, ainsi que les observations d'audience, font émerger des pratiques extrêmement variables selon la juge.

Les rendus de justice des JE et de la cour d'appel compétente pour ces départements confirment une tendance à limiter l'ordonnancement des tests osseux aux cas où un doute est porté sur l'authenticité des documents d'état civil.

La loi, en tout cas c'est comme ça que je raisonne, dit que les tests on peut les ordonner quand il n'y a pas d'actes, et là c'est simple, et face à un document falsifié. Et puis s'il y a des éléments extérieurs qui peuvent faire douter de leur validité, comme dans le cas de la Guinée pour lesquels la PAF estime qu'il y a une fraude généralisée. Alors moi, je vais vous dire, j'ai du mal à vous parler de ça, parce que j'en ai ordonné très peu, et lorsque j'en ai ordonné, j'en tirais tellement peu de chose que j'ai fini par en conclure que je n'en ordonnais pas, tant pis, je faisais sans, parce que ça ne servait à rien. De toute façon, vue l'imprécision qu'il peut y avoir, et même si les médecins légistes nous disent qu'ils prennent en compte la marge d'erreur, ce que j'ai compris c'est qu'on ne peut jamais avoir de certitude entre 17 et 19 ans. (JE 1, TGI 1)

Même lorsqu'un doute subsiste autour de l'«âge vraisemblable " du jeune, certaines juges estiment qu'il est dans leur intérêt de se caler sur la jurisprudence de la cour d'appel, restrictive quant à l'ordonnancement des tests osseux :

Ma position est d'être le plus légaliste possible et d'être au plus près de la jurisprudence de la cour d'appel. Pour éviter aussi de multiplier les recours... moi, ça m'a plombé mon cabinet. J'ai eu 60 MNA qui m'ont saisie entre le $1^{\text {er }}$ septembre et le $1^{\text {er }}$ décembre 2018. (JE 2, TGI 2)

Néanmoins, il se peut aussi que les examens osseux soient ordonnés alors même que les documents sont considérés authentiques par la PAF, et donc parce que l'âge allégué par le jeune est considéré comme invraisemblable. C'est le cas des décisions prises par une des juges du TGI 1 ayant présidé les audiences dont j'ai observé le déroulement fin 2018, qui a été la première à ordonner des scanners de la clavicule au lieu des traditionnelles radiographies du poignet.

Ouais, le doute devrait profiter à l'enfant, c'est ce qu'il dit l'article $47 \mathrm{du}$ code civil, en tout cas c'est la lecture qu'en ont les avocates spécialisées : c'est que si on a un doute, il est mineur. Mais on ne peut pas non plus dire que tout le monde est mineur. Parce qu'on a toujours un doute. On ne peut pas accueillir tout le monde. Il faut vraiment faire la distinction entre ceux qui nécessitent une protection et ceux qui ne la nécessitent pas. (JE 4, TGI 1)

Cette juge a donc fait sien l'argument défendu par le conseil départemental : les ressources manquent, on ne peut pas placer tout le monde seulement parce qu'un doute subsiste. En cas de doute, il n'est pas question qu'il profite à l'enfant (comme le prévoit l'article 388 du code civil) : a minima, le test osseux doit être ordonné, a maxima la prise en charge doit être refusée.

Le problème reste entier dans la mesure où les JE doivent distinguer «ceux qui méritent " de «ceux qui ne méritent pas ». L'arbitrage autour de "cas-limites " cristallise des divergences parfois profondes entre les magistrates. Certaines voudraient limiter la prise en charge aux seuls jeunes "manifestement mineurs" (JE 4, TGI 1), alors que le caractère "manifeste " de la minorité ou majorité ne peut qu'être subjectif et lié à des impressions issues de l'interaction fugace entre la juge et le jeune en situation d'audience.

Voilà, là on est entre nous, le dernier qu'on voit... je lui file beaucoup plus que 16 ans, je veux dire physiquement... je ne sais pas ce que vous en pensez mais... là il a l'air d'avoir plus que 16 ans. Enfin... voilà. Et dans ce cas, le test de la clavicule c'est intéressant pour lui. Parce que, à mon avis, il est plus près de 20 que de $16 \ldots$ (JE 4, TGI 1)

À mes questions sur les éléments qui lui font croire à un âge supérieur à 16 ans, la JE répond succinctement, en évoquant un générique "aspect physique d'adulte » puis l'assurance que, selon elle, le jeune a montré lorsqu'il a pris la parole pendant l'audience. Cette juge se donne en effet pour mission d'exclure davantage d'étrangers aux apparences d'adultes, pour ainsi alléger la pression financière qui s'exerce sur le $\mathrm{CD}$, voire donner un message aux "filières criminelles ". 
À un moment donné, si on dit : "On les accueille tous ", ça veut dire qu'on cautionne la traite des êtres humains quoi. On cautionne les passeurs. On cautionne tous ces parcours qu'ils ont eus... parce qu'ils savent qu'au final ils auront l'accès à l'accueil. Et moi je ne veux pas cautionner ça. [...] Évidemment sur le principe on est tous d'accord, idéalement il faudrait pouvoir accueillir tous les gens qui sont malheureux, mais déjà qu'on a du mal à accueillir nos malheureux, parce qu'on en a aussi en France hein! (JE 4, TGI 1)

Des considérations socio-économiques peuvent donc venir se greffer au raisonnement juridique. Ces arbitrages sont au cœur de la différenciation des pratiques des JE : recourir aux tests ne permet pas de distinguer clairement et sans appel ceux qui sont mineurs de ceux qui ne le sont pas, mais permet d'arbitrer entre les cas, traités "à la chaîne ", de façon à favoriser l'accueil des personnes dont la situation est considérée comme plus compliquée, et donc plus représentative de l'enfance en danger. Les tensions entre juges révèlent donc des visions divergentes de la hiérarchisation des problèmes sociaux à affronter et des objectifs de leur mission. Ainsi, l'indice corporel relevant de l'âge osseux estimé est mobilisé plutôt en dernière instance, lorsque le cas à traiter ne semble pas relever de l'extrême nécessité de prise en charge. C'est à ce contexte qu'il faut rapporter le traitement de la preuve qui n'a donc plus grand-chose à voir avec la question de la vérité de l'âge.

Certaines juges, contrairement à la JE 4, tentent de résister à ces "impressions souvent trompeuses " (JE 1, TGI 1) et se servent d'une interprétation «légaliste " du droit pour écarter l'ordonnancement des expertises médicales. Ainsi au TGI 1, la variété des pratiques au sujet du recours aux tests osseux donne lieu à des tensions entre collègues.

Ce sujet a aussi énormément tendu les relations avec les collègues, et donc notamment avec $\mathrm{M}^{\text {me }}$ (JE 4, TGI 1), puisque moi je lui ai dit que je n'interprétais pas la jurisprudence de la cour d'appel comme elle, et qu'à partir du moment où le jeune a un document, qui en plus a été authentifié, elle ne pouvait pas ordonner l'expertise osseuse, mais c'est ce qu'elle a fait. Vous voyez, c'est une discussion qui pourrait être juridique, parce que ça s'interprète les jurisprudences, mais en fait, ce sujet... on ne peut pas discuter sans finalement opposer un discours qui est politique et qui du coup vient brouiller les cartes. (JE 3,TGI 1)
Ces écarts dans la pratique professionnelle des JE selon leur propension à ordonner ou non les expertises osseuses fondent leur réputation, au sein et en dehors du tribunal :

On ne peut pas en parler, je vous arrête tout de suite. Voilà, c'est à dire les fois où on essaie d'en parler, c'est très... c'est très dur quoi, c'est des positions où on a l'impression que l'on parle plus de politique que de juridisme. Alors en plus, moi je suis membre du syndicat de la magistrature donc nécessairement mon avis làdessus on va l'aborder de façon, voilà... «Elle fait sa gaucho ». Et cetera. Je vous dis que les échanges que l'on peut avoir sur cette question... on va vite me renvoyer à : "Tu défends les migrants ». Voilà, vous voyez ce que je veux dire, non mais, ça vole pas plus haut que ça, je suis désolée! (JE 3, TGI 1)

Des conflits autour de positionnements politiques apparaissent. La variété des décisions autour de l'ordonnancement des tests par le JE tient donc à la fois aux grandes marges d'interprétation des textes, mais surtout, au regard de la fiabilité contestée de ces tests, à la sensibilité politique de chacune des magistrates, qu'elle soit affirmée voire revendiquée (JE 3) ou au contraire non explicitée voire niée.

\section{- Conclusion}

Qualifier une situation, comme l'arrivée de mineurs étrangers ou de migrants en général, d' "urgente " et d' "exceptionnelle " est un acte chargé politiquement : cette rhétorique de l'urgence, combinée avec les moyens de pression dont les conseils départementaux et les préfectures disposent vis-à-vis de la justice, peuvent influencer les décisions des juges des enfants en matière de (non)reconnaissance de la minorité. Leur pouvoir souverain d'appréciation entretient un rapport complexe avec l'État de droit, et de fait contribue à renverser le principe du "doute qui profite à l'enfant " [Slama et Bouix, 2014]. Comme l'avait déjà remarqué Mélanie Gourarier au sujet de l'usage des tests ADN dans le processus de regroupement familial, "la "vérité" établie par les services de l'immigration supplante celle déclarée par les personnes et attestée par les documents d'état civil. Or, la force de cette imposition repose sur une logique du soupçon à l'égard des demandeurs, qui s'inscrit dans une histoire de plus longue durée » 2017 : 52]. Ce doute qui pèse 
à la fois sur le récit du jeune, sur ses papiers, voire sur ses os, véhicule plus fondamentalement une vision du migrant en tant que "fraudeur " et en tant que " coût " [Mitsilegas, 2015].

Les tests osseux fonctionnent ainsi en tant qu'outils de gestion des populations [Makaremi, 2007] que la loi autorise parce que ces mêmes populations sont associées à un phénomène que le gouvernement français présente comme un "problème public ». Les pratiques des JE face à la responsabilité de devoir statuer sur la (non)minorité des jeunes étrangers s'inscrivent en effet dans un contexte socio-politique plus large de «criminalisation des migrants " [Palidda, 1999], où la rhétorique du soupçon et du risque fournit les justifications à la lutte contre la fraude [Huysmans, 2014 : 102-107].

Cette enquête montre précisément qu'un "régime de suspicion » est à l'œuvre [Delmas-Marty, 2009], caractérisé par des glissements successifs des dispositifs juridiques exceptionnels (des radiographies du poignet aux scanners de la clavicule, jusqu'au fichier biométrique pour les mineurs introduit par le décret $n^{\circ} 2019-57$ du 30 janvier 2019) qui vont progressivement légitimer des pratiques relevant de la «frénésie sécuritaire " [ibid. : 470]. Cette dernière "tend à transformer le contrat social en une sorte de contrat d'assurance tous risques qui caractérise les régimes de suspicion : chaque individu est un suspect potentiel» [ibid.]. Ainsi, l'enquête montre quelles sont les postures adoptées par les professionnels de la justice, notamment les juges des enfants, pour hiérarchiser les cas soumis à leur pouvoir décisionnel : peu importe la certitude autour de l'âge chronologique, il faudra décider si le dossier examiné est suffisamment "crédible " et si le jeune "fait " suffisamment enfant pour ordonner sa prise en charge.

Les JE auront plus ou moins tendance à considérer les expertises médicales en tant qu'indices utiles dans la construction de la "crédibilité " de l'âge allégué, même si aucune ne dit avoir une confiance aveugle en leurs résultats. Finalement, ce qui distingue le plus leurs pratiques est la tendance à les ordonner ou non. Étant donné que les résultats des expertises pratiquées par le CHU travaillant avec les TGI 1 et 2 concluent le plus souvent à la majorité, lorsque la juge se donne pour mission de repérer et protéger prioritairement les «manifestement mineurs» elle aura tendance à faire attention au physique et aux attitudes, puis à se méfier des papiers et du récit du jeune, et enfin à ordonner les tests osseux : comme le remarquait à juste titre Chowra Makaremi au sujet des tests ADN en France "[t]out le contraire de la "déclaration sur l'honneur" qui certifie une vérité dans divers moments de la vie administrative, le prélèvement de cellules court-circuite la personne comme être de langage, ne lui reconnaissant nul pouvoir de déclarer, niant l'existence même de quelque chose comme l'honneur dans le jeu aride de l'individu à l'État» [2007]. Le résultat de l'expertise médicale servira alors d'élément de confirmation d'un jugement qui a pris forme au préalable, sur la base d'une impression subjective.

En l'absence d'une expertise médicale capable de " dire vrai » à elle seule, les jeunes sont en effet appelés à convaincre, de par leur comportement et leur aspect physique, de la "crédibilité " de leur récit. Pendant ce parcours vers l'institutionnalisation d'une décision de justice sur l'âge, il n'est pas question pour la juge d'avoir de certitude sur ce qui est vrai ou faux, mais bien de statuer sur ce qui est crédible et cohérent, et ce qui ne l'est pas. Ainsi, ces jeunes sont appelés à être convaincants, à se conformer à ce que l'on attend d'un " enfant en danger ", à la fois par leur aspect et par leur comportement; autrement dit, ils doivent pouvoir être identifiés par les opérateurs de jugement comme potentiellement mineurs : et pour cela, il faut qu'ils "fassent mineurs". $\mathrm{Ou}$ en tout cas, "pas trop majeurs ".

Le test osseux est souvent ordonné par la juge non pas parce qu'elle estime qu'il est porteur d'une vérité irréfutable et qu'il peut être déterminant dans sa décision finale, mais plutôt pour venir confirmer une première impression qu'elle s'est déjà faite au sujet de son âge et parce que son ordonnancement lui permettra de justifier sa décision aux yeux des collègues et des institutions concernées.

À dossiers semblables, le recours aux tests osseux et les rendus de justice peuvent donc différer sensiblement. Surtout, les magistrats qui ont plutôt tendance à s'en méfier, au profit de la présomption de minorité, sont sous pression. La dimension politique de ces luttes judiciaires autour des expertises sur le corps d'autrui est évidente. Face à l'inefficacité de leur capacité à produire une évidence par le corps, les usages des expertises osseuses nous renseignent surtout sur les logiques qui guident les arbitrages d'une justice aux prises avec des logiques institutionnelles et économiques où le soupçon de fraude traverse l'ensemble des étapes de la procédure de reconnaissance de la (non)minorité. 


\section{Notes}

1. L'ensemble des noms a été modifié, pour garantir l'anonymat aux personnes mentionnées dans ce texte.

2. Étant donné que la très grande majorité de jeunes étrangers arrivant dans ces deux départements du sud de la France sont des garçons, j'utiliserai le masculin pour me référer à eux dans ce texte. En revanche, et pour la même raison, j'utiliserai le féminin pour parler des avocates qui défendent leur cause ainsi que des juges des enfants (JE) qui sont censées trancher sur la question de leur minorité.

3. Il s'agit d'observations d'audiences en première instance devant la juge des enfants, saisie par les jeunes via leur avocate, dans le but de contester l'évaluation du DEMI ayant conclu à leur majorité et ayant donc entraîné le refus de les prendre en charge.

4. Je désigne comme "économies morales » des valeurs et des normes, des jugements et des justifications qui sont à l'origine de diverses représentations de la justice et de la vérité, parfois antagonistes, qui s'affrontent ici dans l'arène judiciaire. Voir notamment Fassin et Eideliman, 2012.

5. La question de la construction genrée de l'âge [DIASIO, 2012 ; CHARLAP, 2017] n'est pas abordée ici, puisque le corpus de cas judiciaires étudié est intégralement masculin. Cependant, les entretiens avec les avocates et les juges laissent penser que la condition de fille se déclarant mineure non accompagnée est plus facilement associée à la figure de la "jeune en danger». Cela mériterait sans doute qu'une enquête à part entière y soit consacrée.

6. Mission mineurs non accompagnés, rapport annuel d'activité 2018, juin 2019 [http:// www.justice.gouv.fr/art_pix/RAA-MMNA2018.pdf].

7. Il est important de rappeler que les données sur les MNA se basent sur des estimations, et qu'aucune donnée fiable n'a été produite pour la période antérieure à 2015 en ce qui concerne le nombre de prises en charge en France.

8. Rapport d'information de $\mathbf{M}^{\text {me }}$ Élisabeth Doineau et $M$. Jean-Pierre Godefroy, fait au nom de la commission des affaires sociales, "Mineurs non-accompagnés : répondre à l'urgence qui s'installe ", $\mathrm{n}^{\mathrm{o}}$ 598, 28 juin 2017 [https:// www.senat.fr/rap/r16-598/r16-5981.pdf].

9. [http://www.departements.fr/wp-conten t/uploads/2019/02/Fiche-info-MNA-f\%C3\%A 9vrier-2019.pdf].
10. Le Premier ministre déclarait à ce sujet, lors du Congrès de l'ADF le 20 octobre 2017 «Il faut que l'État prenne à sa charge des choses qui relèvent de compétences plus régaliennes, c'est-à-dire d'une capacité à décider notamment de la majorité ou de la minorité ». Sans que des changements majeurs en la matière aient été validés depuis, l'adoption le 30 janvier 2019 du décret no 2019-57 va dans ce sens, en officialisant la création d'un fichier biométrique national concernant les MNA : l'objectif est de prévenir le détournement du dispositif de protection de l'enfance par des personnes majeures ou des personnes se présentant successivement dans plusieurs départements. La question n'est pas anodine : pour l'instant, le droit commun de la protection de l'enfance est applicable aux MNA au même titre qu'aux nationaux ; transférer à l'État la responsabilité de l'évaluation signifierait renverser le principe de présomption de minorité et de fait, traiter ces jeunes non pas comme des mineurs jusqu'à la preuve du contraire, mais d'abord comme des étrangers dans l'objectif de maitriser les flux migratoires.

11. [https://www.senat.fr/rap/a18-150-7/ a18-150-73.html].

12. Ce qui inclut l'hébergement, l'assistance médicale, l'orientation professionnelle voire la scolarisation pour les moins de 16 ans.

13. Ils sont éducateurs ou assistants sociaux, en principe formés à l'évaluation des MNA et travaillant au sein d'une équipe interdisciplinaire. En principe, parce que si la loi précise que l'instruction des dossiers passe par "des entretiens conduits par des professionnels justifiant d'une formation ou d'une expérience définies par un arrêté des ministres dans le cadre d'une approche pluridisciplinaire et se déroulant dans une langue compréhensible par l'intéressé " (arrêté du 17 novembre 2016), les données de terrain récoltées dans les deux départements étudiés font émerger un très fort turnover des professionnels, une expérience courte dans l'évaluation, et des conditions de travail, liées notamment aux temps réduits pour rédiger et adresser les conclusions des évaluations au PCD, qui limitent les possibilités réelles de conduire des évaluations dans la bienveillance et l'interdisciplinarité.

14. Décret d'application du 24 juin 2016.

15. Les parquets requièrent cette expertise à la demande de la police aux frontières ou de l'Aide sociale à l'enfance. De telles expertises peuvent toutefois être ordonnées par le juge des enfants, qui, contrairement aux procureurs, doivent rencontrer les enfants lors d'une audience afin de les entendre au sujet de leur parcours. C'est sur ce cas de figure que l'enquête dont il est question ici s'est concentrée. Voir RONGÉ 2016.

16. Il est important de préciser que les services de la PAF rendent un "avis", favorable ou défavorable, après l'expertise des documents analysés : en effet, ils travaillent à partir de modèles d'acte, et ils ne procèdent pas à des analyses plus poussées impliquant de se mettre en contact avec les services émetteurs des documents. Ils ne peuvent travailler qu'en fonction des références dont ils disposent, ce qui fait que dans de nombreuses situations, ils ne peuvent pas s'exprimer avec certitude : c'est le cas des documents d'état civil guinéens (Conakry), pour lesquels un "avis défavorable " est rendu systématiquement par la PAF à cause du soupçon de "fraude généralisée ", alors que les services ne sont pas pour autant en mesure de se prononcer sur leur authenticité au cas par cas.

17. Il correspond au stade de maturation du squelette. Sa détermination est basée sur l'analyse de l'activité des cartilages de conjugaison, visible sur les épiphyses.

18. Sans vouloir être exhaustive, le Haut conseil de la santé publique [2014], l'Académie nationale de médecine [2007], le Comité national consultatif d'éthique [2005], la Commission nationale consultative des droits de l'homme [2014], le Défenseur des droits [2015, 2017], ont émis un avis défavorable à la pratique de ces tests. Ces instances en ont signalé le caractère violent et offensant pour les personnes, souligné l'inadaptabilité à des fins judiciaires, la grande incertitude de résultats quant à la détermination d'un âge chronologique, surtout concernant les mineurs à partir de 15 ans (la marge d'erreur est alors de 1 à 3 ans). Il a été en outre établi que les interprétations des clichés radiographiques ou des scanners sont également sujettes à des écarts importants selon les praticiens et les unités médico-légales qui les réalisent. Pour toutes ces raisons, l'Association des médecins britanniques et celle des dentistes britanniques ont pris collectivement position déjà en 2007 contre ces pratiques chez les jeunes se déclarant mineurs non accompagnés.

19. Ces éléments ont été au centre d'une question prioritaire de constitutionnalité (QPC) en 2019 : à la suite d'un pourvoi formé devant la Cour de cassation au sujet de l'usage de ces tests et de leur rôle dans le refus de prise en charge d'un jeune par l'ASE, la Cour a décidé, en date du 21 décembre 2018, de transmettre la QPC au Conseil constitutionnel pour qu'il s'exprime sur leur interdiction éventuelle. Avec la décision $\mathrm{n}^{\circ}$ 2018-768 QPC du 21 mars 2019 , le Conseil décrète que les «deuxièmes et troisièmes alinéas de l'article 388 du code civil, dans sa rédaction résultant de la loi ${ }^{\circ}$ 2016297 du 14 mars 2016 relative à la protection de l'enfant, sont conformes à la Constitution" : autrement dit, le recours aux tests osseux est entériné.

20. Mission mineurs non accompagnés, Rapport 2018, op. cit. 


\section{Références bibliographiques}

BAILleul Corentin, 2017, «Publication d'un rapport d'information du Sénat relatif à la prise en charge (au rabais) des mineurs non accompagnés. Commentaire critique du rapport d'information du Sénat 28 juin 2017 sur la prise en charge sociale des mineurs non accompagnés ", Journal du droit des jeunes, $8: 146-152$.

Bailleul Corentin et Daniel SenOvilla Hernandez, 2016, Dans l'intérêt supérieur de qui ? Enquête sur l'interprétation et l'application de l'article 3 de la Convention Internationale des Droits de l'Enfant dans les mesures prises à l'égard des mineurs isolés étrangers en France, Poitiers, MIGRINTER, Rapport MINAS.

BARRETT Kimberly et William GEORGE, 2005, Race, Culture, Psychology, and Law, Thousand Oaks, Sage.

CARAYON Lisa, Julie MATtiUSS et Arthur VuATtOUX, 2018, " "Soyez cohérent, jeune homme !" : Enjeux et non-dits de l'évaluation de la minorité chez les jeunes étrangers isolés à Paris ", Revue française de science politique, 68 (1) : 31-52.

CARAYON Lisa, Julie MATtiUssi et Arthur VuAtTOUX, 2020, «Étrange jeunesse, jeunesse étrangère. Formes de culturalisme dans l'évaluation des jeunes isolés étrangers ", Agora débats/jeunesse, 84 (1) : 109-124.

CHARIOT Patrick, 2010, «Quand les médecins se font juges : la détermination de l'âge des adolescents migrants ", Chimères, 74 (3) : 103-111.

CHARlAP Cécile, 2017, "L'âge, le genre et la classe au cœur de la physiologie. Retour sur une enquête auprès de femmes ménopausées ", SociologieS [http://journals.openedition.org/ sociologies/5994, mis en ligne le 21 février 2017, consulté le 10 août 2019].

COllectif CeTte FranCE-LÀ, 2010, «La technologie du soupçon : tests osseux, tests de pilosité, tests ADN », Mouvements, $62: 80-83$.

DELMAS-MARTy Mireille, 2009, «Libertés et Sûreté. Les Mutations de L'État de Droit ", Revue de Synthèse, Springer Verlag/Lavoisier, 130 (3) : 465-491.

DiAsIO Nicoletta, 2012, "Maillage des temps et gouvernement des corps dans la construction des rapports d'âge et de genre ", SociologieS [http://journals.openedition.org/sociologies/4118, mis en ligne le 15 novembre 2012, consulté le 10 juillet 2019].

DiAsIO Nicoletta, 2014, «Repenser la construction des âges : sortie de l'enfance et temporalités plurielles ", Revue des sciences sociales, $51: 16-25$.

DiAsio Nicoletta (dir.), 2015, "Grandir, pouvoirs et périls », Ethnologie française, 45 (4).

DiAsIO Nicoletta et Virginie VINEL, 2017 (dir.), Corps et préadolescences. Intimes, privés, public, Rennes, PUR.

DODIER Nicolas, 1991, "Expérience privée des personnes et expertises médico-administratives. Une enquête dans la médecine du travail ", Sciences sociales et santé, 9 (2) : 79-121.

DODIER Nicolas, 1993, L’Expertise médicale. Essai de sociologie sur l'exercice du jugement, Paris, Métailié.

FASSIN Didier et Jean-Sébastien EIDELIMAN (dir.), 2012, Économies morales contemporaines, Paris, La Découverte.
FASSIN Didier, Yasmine BOUAGGA, Isabelle COUTANT et al., 2013, Juger, réprimer, accompagner. Essai sur la morale de l'État, Paris, Seuil.

FOCARDI Martina, Federica DE LUCA, Gian ARISTIDE NORELLI et al., 2014, "Age estimation for forensic purposes in Italy: ethical issues ", International Journal of Legal Medicine, 128 (3): 515-522.

GISTI, 2017, L'Accès aux droits et aux soins des mineurs non accompagnés en France [https://www.gisti.org/IMG/pdf/guide_ mna-mdm_partenaires.pdf].

GOURARIER Mélanie, 2017, "Faire la frontière dans les murs du laboratoire. Destins migratoires et usages de l'ADN aux États-Unis », Genèses, 108 (3) : 48-68.

Greulich William W. et Sarah Idell PyLE, 1959, Radiographic atlas of skeletal development of the hand and wrist, Stanford, Stanford University Press.

Hassenteufel Patrick, 2010, "Les processus de mise sur agenda : sélection et construction des problèmes publics ", Informations sociales, 157 (1) : 50-58.

Hermetet Coralie, Pauline SAInT-Martin, Arsène GamBIER et al., 2018, "Forensic age estimation using computed tomography of the medial clavicular epiphysis: a systematic review ", International Journal of Legal Medicine, 132 (5) : 14151425.

Human Rights WatCH, 2018, "C'est la loterie". Traitement arbitraire des enfants migrants non accompagnés à Paris " [https://www.hrw.org/fr/report/2018/07/04/cest-laloterie/ traitement-arbitraire-des-enfants-migrants-non-accompagnesparis].

HuYsmans Jef, 2014, Security Unbound: enacting democratic limits, Londres, Routledge.

IGA, IGAS, IGJ et ADF, 2018, Rapport de la mission bipartite de réflexion sur les mineurs non accompagnés, La Documentation française, 15 février, 51-52 [https://www.ladocumentationfrancaise.fr/var/storage/rapports-publics/184000336.pdf].

LAMARCHE-VADEL Gaëtane, 2016, "Tests osseux pour les mineurs étrangers isolés ", Multitudes, 63 [http://www.multitudes.net/tests-osseux-pour-les-mineurs-etrangers-isoles/].

MAKAREMI Chouwra, 2007, " "De l'esprit des lois". Quelques éléments de réflexion à propos de la "loi ADN" en $\begin{array}{llll}\text { France ", } & \text { Eurostudia, } & 3 & \text { (2) }\end{array}$ id.erudit.org.inshs.bib.cnrs.fr/iderudit/071838ar10.720/ 017839ar].

MÉDECINS DU MONDE, 2017, «Argumentaire relatif à l'interdiction des méthodes médicales aux fins de détermination de l'âge des mineurs non accompagnés " [https:// www.infomie.net/IMG/pdf/argumentaire_medecins_du_ monde_determination_medicale_de_1_age_30_11_dif.pdf].

MÉDECINS DU MONDE, 2019, "L'accueil des migrants, reflet d'une crise de solidarité " [https://www.medecinsdumonde.org/fr/actualites/tribunes/2019/01/15/laccueil-desmigrants-reflet-dune-crise-de-solidarite].

MitsiLegas Valsamis, 2015, The Criminalisation of Migration in Europe. Challanges for Human Rights and the Rule of Law, Londres, Springer.

NOLL Gregor, 2016, "Junk science? Four arguments against the Radiological Age Assessment of Unaccompanied Minors Seeking Asylum ", International Journal of Refugee Law, 28 (2) : 234-250. 
ONTELl Francesca, Marija IVANOVIC, Deborah S. ABLIN et al., 1996, "Bone age in children of diverse ethnicity ", American Journal of Roentgenology, 167 (6) : 1395-1398.

PALIDDA Salvatore, 1999, "La criminalisation des migrants ", Actes de la recherche en sciences sociales, 129 : 39-49.

PERROT Adeline, 2016, "Devenir un enfant en danger, épreuves d'âge et de statut : le cas «limite " des mineurs isolés étrangers en France », Agora débats/jeunesses, 74 (3) : 119-130.

Plate-FORME MineURs EN ExIL, 2017, «L'estimation de l'âge des MENA en question : problématique, analyse et recommandations » [http://www.mineursenexil.be/files/Image/ mena-Cadre-juridique/Estimation-de-l-age-asprinted.pdf].

Prout Alan, 2000, The Body, Childhood and Society, Basingstoke, Macmillan.
PRUvOST Marie-Odile, Cyril BORAUD et Patrick CHARIOT, 2010, "Skeletal age determination in adolescents involved in judicial procedures. From evidence-based principles to forensic practice ", Journal of Medical Ethics, 36 : 71-74.

RONGÉ Jean-Luc, 2016, "Les "mineurs isolés étrangers" (MIE) deviennent des "mineurs non accompagnés" (MNA) Que tout change... pour que rien ne change!", Journal du droit des jeunes, 358-359-360 (8) : 20-42.

SAINT-MARTIN Pauline, 2014, Apport de l'imagerie par résonnance magnétique dans la détermination de l'âge chez le sujet vivant, thèse de doctorat en médecine, université de Toulouse 3.

SLAMA Serge et Anita BouIX, 2014, "Mineurs isolés étrangers : la fabrique d'un infra-droit d'exclusion ", Actualité juridique Famille, Dalloz, 84.

VAILlY Joëlle et Gaëlle KRIKORIAN, 2018, " Durabilité et extension du soupçon. Catégorisations et usages policiers du fichier d'empreintes génétiques en France ", Revue française de sociologie, 59 (4) : 707-733.

\section{ABSTRACT}

Minor to the bone?

The role of juvenile court judges in determining the age of unaccompanied migrant minors using bone testing

This article analyses the role of skeletal examinations carried out during the judicial process to determine whether unaccompanied migrants are indeed minors. The study focuses on discussions on their capacity to "tell the truth" of an individual's chronological age that emerge during and outside hearings of the Children's Courts. Based on qualitative data collected during ethnographic field work in the south of France, the article discusses the controversies surrounding the legitimacy and reliability of these "technologies of suspicion", highlighting the political dimension of juridical practices that involve expert assessments of an individual's body.

Keywords: Unaccompanied minors. Bone age. Age assessment. Juvenile court judge. Technologies of suspicion

\section{ZUSAMMENFASSUNG}

Minderjährig bis auf die Knochen?

Jugendrichter und das Alter des jungen Ausländers durch das Prisma der Knochenprüfung.

In diesem Beitrag wird der Platz der Knochenprüfung im Prozess der (Nicht-)Anerkennung der Minderjährigkeit des isolierten jungen Ausländers analysiert. Der Artikel konzentriert sich insbesondere auf die Debatten über ihre Fähigkeit, „die Wahrheit“" über das Alter von Jugendlichen auzusagen. In den untersuchten Fällen stehen die Jugendlichen gerade vor dem Jugendrichter oder kurz vor der Anhörung. Auf der Grundlage von Material, das im Rahmen einer in zwei französischen Städten durchgeführten ethnographischen Erhebung gesammelt wurde, analysiert der Kern des Beitrags die Debatten um die Legitimität und Zuverlässigkeit dieser „Technologien des Verdachts“ und beleuchtet die politische Dimension der gerichtlichen Praktiken im Zusammenhang mit Gutachten über die Körper anderer.

Schlagwörter: Unbegleitete Minderjährige. Knochengutachten. Altersbestimmung. Jugendrichter. Verdachtstechnologien.

\section{RESUMEN}

Menor hasta los huesos?

El juez de menores y la edad del joven extranjero bajo el prisma de los análisis óseos.

Esta contribución propone analizar el protagonismo de los análisis óseos en el proceso de (no)reconocimiento de un menor extranjero no acompañado. El artículo se centra en los debates relativos a su capacidad para "decir la verdad" sobre la edad de estos jóvenes durante las audiencias y al margen de ellas ante el juez de menores. Basándose en el material recogido a través de una encuesta etnográfica realizada en dos ciudades francesas, la esencia del artículo es el análisis de los debates sobre la legitimidad y la fiabilidad de esas "tecnologías de la sospecha" y pone de relieve la dimensión política de las prácticas judiciales en torno a los dictámenes periciales sobre el cuerpo ajeno.

Palabras clave: Menores no acompañados. Análisis óseos. Determinación de la edad. Juez de menores. Tecnologías de sospecha. 